\title{
FOM-Edition
}

FOM Hochschule für Oekonomie \& Management

Weitere Bände in dieser Reihe

http://www.springer.com/series/12753 
Die FOM-Edition wird herausgegeben von FOM Hochschule für Oekonomie \& Management. 
Friederike Müller-Friemauth • Rainer Kühn

Silicon Valley als

unternehmerische

Inspiration

Zukunft erforschen - Wagnisse eingehen Organisationen entwickeln

Springer Gabler 
Friederike Müller-Friemauth FOM Hochschule für Oekonomie

\& Management

Köln

Deutschland
Rainer Kühn

kühn Denken auf Vorrat

Odenthal

Deutschland

Dieses Werk erscheint in der FOM-Edition, herausgegeben von FOM Hochschule für Oekonomie \& Management.

FOM-Edition

ISBN 978-3-658-09328-0

DOI 10.1007/978-3-658-09329-7
ISBN 978-3-658-09329-7 (eBook)

Die Deutsche Nationalbibliothek verzeichnet diese Publikation in der Deutschen Nationalbibliografie; detaillierte bibliografische Daten sind im Internet über http://dnb.d-nb.de abrufbar.

\section{Springer Gabler}

(C) Springer Fachmedien Wiesbaden 2016

Das Werk einschließlich aller seiner Teile ist urheberrechtlich geschützt. Jede Verwertung, die nicht ausdrücklich vom Urheberrechtsgesetz zugelassen ist, bedarf der vorherigen Zustimmung des Verlags. Das gilt insbesondere für Vervielfältigungen, Bearbeitungen, Übersetzungen, Mikroverfilmungen und die Einspeicherung und Verarbeitung in elektronischen Systemen.

Die Wiedergabe von Gebrauchsnamen, Handelsnamen, Warenbezeichnungen usw. in diesem Werk berechtigt auch ohne besondere Kennzeichnung nicht zu der Annahme, dass solche Namen im Sinne der Warenzeichenund Markenschutz-Gesetzgebung als frei zu betrachten wären und daher von jedermann benutzt werden dürften. Der Verlag, die Autoren und die Herausgeber gehen davon aus, dass die Angaben und Informationen in diesem Werk zum Zeitpunkt der Veröffentlichung vollständig und korrekt sind. Weder der Verlag noch die Autoren oder die Herausgeber übernehmen, ausdrücklich oder implizit, Gewähr für den Inhalt des Werkes, etwaige Fehler oder Äußerungen.

Gedruckt auf säurefreiem und chlorfrei gebleichtem Papier

Springer Fachmedien Wiesbaden ist Teil der Fachverlagsgruppe Springer Science+Business Media (www.springer.com) 
Wir müssen das Silicon Valley nicht kopieren, aber wir müssen es kapieren.

Josef Kaeser, Siemens AG 


\section{Vorwort}

Neben die weitreichenden wirtschaftspolitischen Folgen der monetären Krisen seit 2008 hat sich ein weiteres Thema in den Vordergrund der ökonomischen Lageberichte geschoben: Das für viele (gerade im „Alten Europa“) erstaunliche Wirtschaftsgeschehen im Silicon Valley. Kalifornische Unternehmen, ehemals Garagen-Experimente exzentrischer Nerds, schicken sich an, die Weltwirtschaft zu dominieren.

Wer sich die weltweiten Kommentare zum kalifornischen Traum genau anschaut, erkennt schnell, dass Kenntnisse über das Silicon Valley erstaunlich oft oberflächlich sind - trotz der vielen Pilgerreisen ins ökonomisch gelobte Land, die zahlreiche Unternehmer in der letzten Zeit antraten. Urteilskriterien für die dortige ökonomische Praxis kommen ausgesprochen „klassisch“ daher. Maßstäbe sind zumeist konventionelle betriebswirtschaftliche - also kanonisch gewordene - Kriterien, um ökonomisches Handeln zu bewerten. Sind die Strategien dort konsistent und robust, die Akquisitionen dazu passend? Wie sehen die Ziele aus, wird (dem hiesigen Verständnis nach) professionell gemanagt und kontrolliert?

Was aber, wenn all diese Maßstäbe gar nicht die ökonomischen Hauptkriterien der Kalifornier träfen? Wenn Google, Facebook, Apple, Amazon und Co. nicht trotz dieser Distanz zum etablierten Regelkanon, sondern gerade deswegen solch immensen Erfolg hätten?

Wir wollen mit unserem Buch den kalifornischen Traum begreifbar machen. Allgemeiner gesagt: Wir interessieren uns für alternative Ökonomieleitbilder, die es innerhalb der sich stetig weiter global vernetzenden Wirtschaft zuhauf gibt. Genug jedenfalls, um das eigene ökonomischen Handeln an ihnen zu überprüfen.

Dazu fundieren wir einleitend (Kap. 1) unser Verständnis ökonomischer Praxis. Aus dieser Position folgt letztlich ein - für europäische Verhältnisse ungewöhnlicher - Wechsel: Die Theorie wird konsequent in den Dienst der Praxis gestellt (amerikanisches Wissenschaftsverständnis, „Pragmatismus“). Der entscheidende Vorteil: Die theoretische Perspektive steht nicht neben beziehungsweise außerhalb der Praxis. Das ökonomische Geschehen im Silicon Valley kann mit dessen eigener pragmatischer Vorgehens- und Sichtweise beschrieben werden. Wir setzen uns - und Ihnen - also quasi die gleiche Brille auf, mit der die Kalifornier die Welt betrachten. 
Danach beschäftigen wir uns damit, wie in Silicon-Valley-Unternehmen Entscheidungen hoher Trag- und Reichweite zustande kommen (Kap. 2). Die Beschreibung hält sich strikt an Vorbilder; Meister ihres Fachs, denen wir abschauen, wie sie Entscheidungen in ihrer Organisation herbei,,führen“.

Im Anschluss geht es darum, wie diese Unternehmen exzellent geworden sind (Kap. 3 ). Wie sie unternehmerischen Erfolg nicht durch exakte Planung - ein Denken vom Heute ins Morgen -, sondern durch gedankliche Vorwegnahmen realisieren. Dabei geht es um ein reflexives Spiel mit Zeit: Um gedanklich kontrollierte Vorgriffe und Rückgriffe, Antezipation und Erinnerung, Zukunftsbilder und Lehren aus der Vergangenheit. Zeitmodelle sind zwar kulturell geprägt und beeinflussen Denken und Handeln nur latent; man kann sie jedoch ökonomisch binden und bearbeiten - und zu Wirtschaftszwecken nutzen. Methoden und unterschiedliche Soziokulturen (auch neben der kalifornischen), die genau dies tun, stellen wir vor. In einem Land, das qua Ursprungsmythos nichts anderes unternimmt, als diejenige Vorstellung wirklich werden zu lassen, die einst die Gründer der Vereinigten Staaten von Amerika imaginiert haben (American Dream), ist ein solcher Umgang mit Zeit längst nicht so kontraintuitiv, wie er für uns Europäer auf den ersten Blick erscheint. Ganz im Gegenteil: Er begründet in der Neuen Welt eine alternative Form sozial gehaltvoller Ökonomie.

Abschließend unterbreiten wir einen Vorschlag zur Profilierung dieses Ökonomieleitbildes, das sich im Lichte des kalifornischen Wegs abzeichnet. Es entstammt zwar einem hochspeziellen soziokulturellen Klima, nämlich demjenigen des Silicon Valley, und ist deshalb nicht einfach kopierbar. Aber: Vom Valley kann man sich inspirieren lassen. Es zeigt einen - zugegeben irritierenden, für uns fremdartigen - Weg auf, wie sich Wirtschaft auch verstehen lässt. Es birgt Potenziale für viele Teile der Welt; Potenziale, die von der kalifornischen, für unsere Augen oft kruden und sozialethisch fragwürdigen Soziokultur leicht überdeckt werden. Um diese Kraftreserven, Leistungsvermögen und Wirkungsfähigkeiten geht es uns. Denn auch, wenn uns Europäern einige Werte dieses Ökonomieleitbildes nicht gefallen: Die zukunftsemphatische unternehmerische Denkart, auf der es fußt, steht im Prinzip jedem Unternehmen offen. Zukunft als Passion - um diesen Kern dreht sich kalifornisches Denken. Um seine Kraft zu nutzen, muss man es jedoch verstehen.

Auf dem Weg nachzuvollziehen, was die kalifornische Ökonomie antreibt, gerät man allerdings an die Grenzen der Erklärungskraft rein betriebswirtschaftlichen Wissens. Wir bedienen uns daher der Instrumente der Zukunftsforschung: des Wissenschaftsverständnisses, der Methoden und der zentralen Forschungsperspektive. Diese Disziplin ist in Kalifornien in der ersten Hälfte des letzten Jahrhunderts entstanden. Innerhalb der Betriebswirtschaftslehre ist der Rückgriff auf „Future Sciences“ zwar noch ungewöhnlich, aber auch nicht mehr völlig neu; erste Anstrengungen, zukunftsforscherisches Denken an die ökonomischen Wissenschaften anzuschließen, sind gemacht, wenn auch hierzulande noch spärlich. Wir möchten zeigen, wie fruchtbar diese transdisziplinäre Perspektive ist, und wie lehrreich und instruktiv globales Wirtschaften, das auf der Höhe der zeitgemäßen wissenschaftlichen Erkenntnisse bearbeitet wird, sein kann. Das Zusammenrücken der Welt 
ist ein Innovationsturbo zur Mehrung intellektuellen Kapitals. Im Land der Dichter und Denker könnte diese Einsicht eine Menge bedeuten; vielleicht sogar bewirken.

Wir danken der FOM Hochschule für Oekonomie \& Management, insbesondere Herrn Professor Thomas Heupel und Herrn Dipl.-jur. Kai Enno Stumpp, für die Aufnahme des Werkes in die FOM Edition und für die Begleitung bei dessen Erstellung.

Odenthal, im Dezember 2015 


\section{Inhaltsverzeichnis}

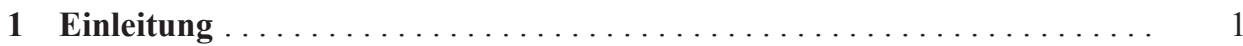

1.1 Die Perspektive einer sozialwissenschaftlich informierten Ökonomie ... 7

1.1.1 Sachliches Problem: Komplexitätsüberlastung .......... 7

1.1.2 Wirtschaftswissenschaftliches Problem: Überkommener

Universalismus . .......................... 9

1.1.3 Entscheidungsproblem: Inflexibilität $\ldots \ldots \ldots \ldots \ldots \ldots \ldots, 10$

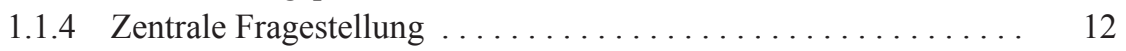

1.2 Theoriepolitische Grundentscheidungen $\ldots \ldots \ldots \ldots \ldots \ldots \ldots \ldots . \ldots \ldots$

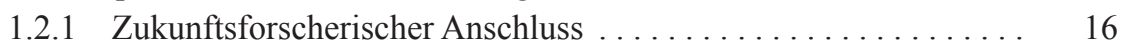

1.2.2 Betriebswirtschaftlicher Anschluss $\ldots \ldots \ldots \ldots \ldots \ldots \ldots . \ldots 18$

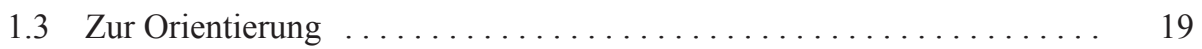

Literatur $\ldots \ldots \ldots \ldots \ldots \ldots \ldots \ldots \ldots \ldots \ldots \ldots \ldots \ldots \ldots \ldots \ldots \ldots \ldots \ldots, 21$

2 Entscheiden - Die unternehmerische Identität festlegen $\ldots \ldots \ldots \ldots .23$

2.1 Kalifornisches Unternehmertum ...................... 25

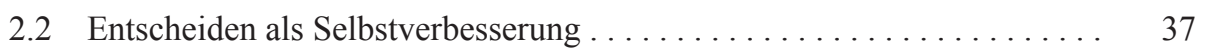

2.2 .1 Tiefenkompetenz: Deep Play . . . . . . . . . . . . . . . 38

2.2.2 Meinungsstreit: Straight Talk ..................... 44

2.2 .3 Umfeldkompetenz ......................... 46

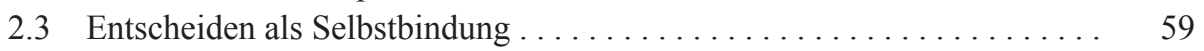

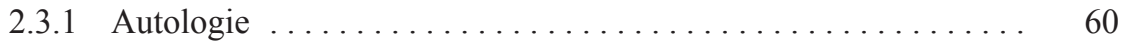

2.3.2 Lernen und Temporalisieren $\ldots \ldots \ldots \ldots \ldots \ldots \ldots \ldots . \ldots 6$

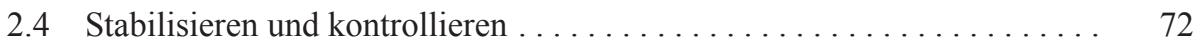

2.4.1 Semantischer Wechsel . . . . . . . . . . . . . . . . . . . . . . . 74

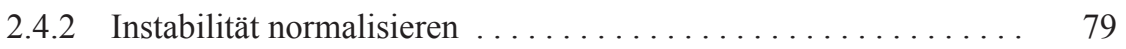

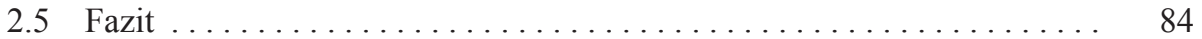

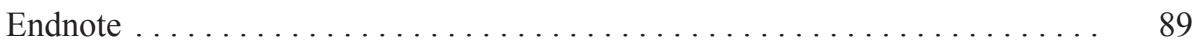

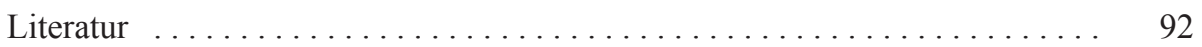


3 Exzellent werden - Unternehmen entwickeln . . . . . . . . . . . . . 95

3.1 Antezipieren: Das Erfassen von Zukunft in der unternehmerischen

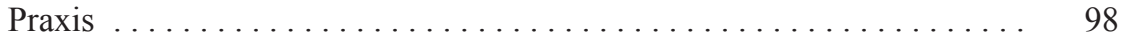

3.1.1 Der praktische Umgang mit Zeit: Entbergen und Projizieren . . . . 99

3.1.2 Kognitiver Umgang mit Zeit: Sich begeistern und engagieren . . . 105

3.2 Methodenbeispiele ......................... 112

3.2.1 Grundprinzip der Zukunftsforschung . . . . . . . . . . . . . 113

3.2.2 Szenarien: Planen und Entscheiden in Alternativen . . . . . . . . . 119

3.2.3 Backcasting: Futur-II-Schocks . . . . . . . . . . . . . . . . 123

3.2.4 Abduktion: Logisch neue Regeln ableiten . . . . . . . . . . . . . . . . 128

3.3 Vier Innovationstypen exzellenzorientierter

Unternehmensentwicklung ...................... 136

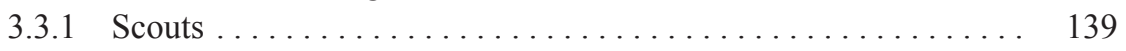

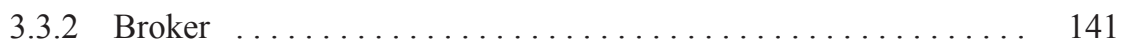

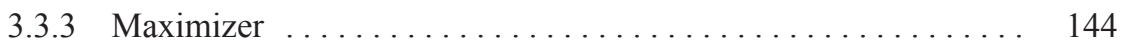

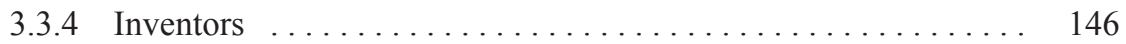

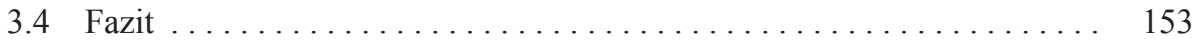

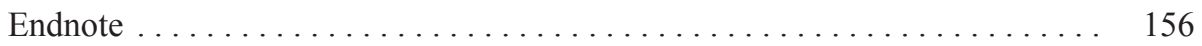

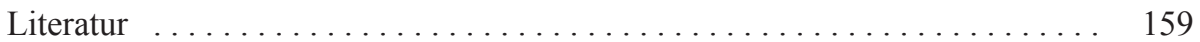

4 Zukunftsintelligenz-Grundlagen einer Preconomics* . . . . . . . . . 163

4.1 Zukunftsentscheidungen: Unternehmensbeispiele ... . . . . . . . . . 164

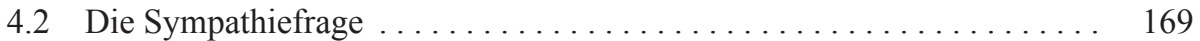

4.3 Werkzeugkiste europäischer Scouts . . . . . . . . . . . . . . . . . . 174

4.4 BWL beim Wort genommen $\ldots \ldots \ldots \ldots \ldots \ldots \ldots \ldots \ldots \ldots \ldots \ldots$

Endnote . . . . . . . . . . . . . . . . . . . . . . . 180

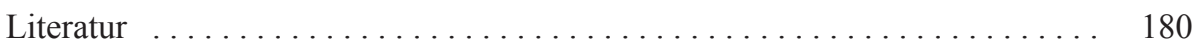




\section{Über die Autoren}

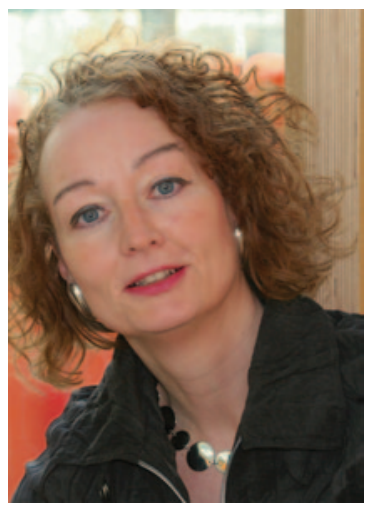

Dr. Friederike Friemauth ist promovierte Sozialwissenschaftlerin. Sie arbeitete mehrere Jahre für die Zukunftsforschungsabteilung der damaligen DaimlerChrysler AG, Berlin, und leitete die Trendforschung bei SinusSociovision, Heidelberg. Heute ist sie Professorin für Allgemeine Betriebswirtschaftslehre, strategisches Marketing und Innovationsmanagement an der FOM Hochschule für Oekonomie \& Management, Köln.

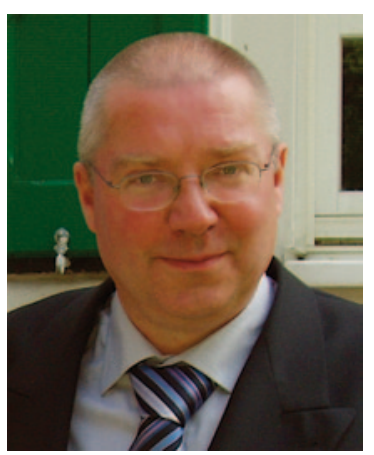

Dr. Rainer Kühn ist promovierter Sozialwissenschaftler. Er war über mehrere Jahre Dozent am politikwissenschaftlichen Institut der FU Berlin, in der Erwachsenenbildung und als stellvertretender Geschäftsführer einer Mediendienstleistungsagentur tätig. Heute ist er freier Publizist.

Beide sind Inhaber von KÜHN DENKEN AUF VORRAT, Odenthal bei Köln. 